\title{
Agent Technology in Supply Chains and Networks An exploration of high potential future applications
}

Jos van Hillegersberg and Hans Moonen

\author{
Department of Decision and Information \\ Sciences, Rotterdam School of Management, \\ Erasmus University Rotterdam \\ F1-27, PO Box 1738, 3000 DR, Rotterdam, \\ The Netherlands, \\ Email: \{jhillegersberg,hmoonen\}@fbk.eur.nl \\ Phone: +31-10-4082624
}

\begin{abstract}
This paper reports on an ongoing research project that is aimed at evaluating how software agents can improve performance of supply chains and networks. To conduct this evaluation, first a framework is developed to classify potential applications of software agents to supply networks. The framework was used in workshop sessions with logistics and information systems experts from industry, software/consultancy and academia to identify promising areas for agents. Based on the framework and the outcome of the workshop sessions, this paper presents promising application areas for the near future and beyond.
\end{abstract}

\section{Introduction}

As competition increases and product life cycles shorten, traditional static supply chains are no longer sufficient. More and more organizations focus on collaboration in supply chains and networks of organizations to achieve a competitive advantage. In such organizational networks, dense and tightly coupled activities increase the difficulty and costs of coordination. Moreover, as tasks become more accumulated of individual contributions and become more contingent upon each other, actors need to connect and share information and knowledge. Collaboration, complex coordination and information sharing between organizations require effective support through information systems.

Software agent technology has been studied for over a decade in academic and research settings, e.g. see [9]. Several authors have identified potential isolated areas within logistics networks were agent could be applied. For example, Khoo et al. [4] describe the potential use of agents to automating part-procurement on the web. However, actual applications to logistics networks are only beginning to appear and their broad potential use in

\author{
Thierry Verduijn and Justus Becker
}

\author{
TNO Inro \\ Schoenmakerstraat 97, \\ PO Box 6041,2600 JA, Delft, The Netherlands, \\ Email: \{tmv, j.becker\}@inro.tno.nl, \\ Phone: +31-15-2694884
}

supporting supply chains has only recently received attention. Some promising technologies, tools, frameworks, case studies and the first commercial applications have appeared over the last few years that could accelerate the adoption of agent technology in the B2B logistics domain. However. there is no comprehensive overview available. Thus, potential users of agent technology to support logistics networks do not have a tool available to assess the various application areas and to identify were agent technology is likely to bring highest value. Therefore, the main objective of this research is to identify potential opportunities for software agent technology to support current and future supply chains/networks.

Within the domain of supply chain and network applications, this study focuses on inter-organizational processes as opposed intra-organisational processes. Interorganizational coordination processes increasingly require IT support and are usually not covered sufficiently by traditional information systems such as legacy systems, Supply Chain Management systems and ERPs. Although more recently E-markets and E-Hubs have been introduced that also aim at supporting inter-organizational coordination, their success has been limited. They mainly provide connectivity, information exchange and support for trading between companies in a network. By increasing transparency through listing supply and demand, these systems have often introduced fierce price competition instead of collaboration. As a result, many organizations have been reluctant to use these systems. Furthermore, even after these systems are successfully introduced, timeintensive and operational-level decisions have to be made by human actors, resulting in high costs and limited benefits. In contrast, applying software agents to support supply chain and network coordination could potentially provide more added value.

In a multi-agent system that supports B2B coordination in supply chains, several types of agents with various roles 
could be deployed, e.g. agents that support business activities (searching, negotiating, monitoring, scheduling,...), agents that support human work (searching information, support decisions, ...) and agents that provide system level activities (connectivity, security, business transaction management, interoperation,...). Agents are especially useful as intermediary software, as they can processes information from specific resources to control supply chain processes [5].

Agents thus do not only enable connectivity in a logistics network, but in addition enable decentralised control of B2B processes. The central research question therefore is: In which inter-organizational logistic processes are intelligent agents expected to deliver added value?

To address this question, we develop a framework that focuses on B2B coordination processes and includes supply chains as well as network configurations (section 2 ). In section 3 , we use this framework as a basis for identifying promising areas in a workshop with academic and industry representatives (section 4). The final section presents the main conclusions and addresses future research.

\section{A framework for agents in supply networks}

In recent years several scholars have published on the application of multi-agent systems in supply chains. Timm et al. [8] classify on-going research into two main streams. One stream is aimed at creating intelligent agents on a theoretical level in order to develop, refine and evaluate various concepts, methods and techniques. Within the second stream, multi-agent systems are used for modelling, managing, controlling and simulating information flows within a single company or networks of companies, e.g. logistics networks on a more applicationoriented level. The latter research stream serves the objective of this research; therefore we first review the wide body of literature in this area, to understand the current state-of-art in application-oriented theory.

Yuan et al. [10] present a framework for application agent technology in supply chain collaboration. Collaboration between organisations can be defined on different levels: collaborative information sharing; collaborative operation (planning of operations) and collaborative configuration (design of the supply chain). Agent technology can be applied to each of these levels. In their review of academic literature they distinguish four groups of proposed agent applications for supply chain management to classify these contributions: (1) General framework for integration and cooperation; (2) Agent systems with specific focus; (3) Supply chain formation; and (4) Agent-based modelling simulation. This framework, though useful at a high level, does not provide enough detail for an analysis of a specific B2B supply chain

Scholz-Reiter \& Höhns [7] propose an agent-based framework for supply chain collaboration based on the SCOR (Supply Chain Operations Reference) model. Their approach is of a conceptual nature and does not present practical examples or a detail description of the supply chain processes that can be supported by agents.

Blake [1] argues that agent capabilities and opportunities are not one-to-one with individual business processes but that agent capabilities are most effective when applied to a grouping of the phases. He then identifies four groups: (1) strategic sourcing; (2) electronic procurement; (3) workflow and supply chain management; and (4) supplier relationship management. Since this framework focuses mainly on sourcing, it is too limited for our purpose.

As none of the frameworks identified meets our goals, we develop a framework that starts from supply chain coordination and attempts to identify how and why agent technology can provide an added value to supply chain coordination. For the definition of the framework for intelligent agent applications in supply chains we define socalled operating environments. Operating environments describe a specific situation within logistics networks in which several supply chain partners have to coordinate their processes. To define the operating environments, we adopt the different settings of inter-organizational collaboration (first dimension of the framework) - mainly based upon the classification of Inter-organizational systems by Hong and Kim [3] - and combine them with supply chain process approach used by Scholz-Reiter \& Höhns [7] (second dimension of the framework).

The first dimension consists of the type of coordination. Hong and Kim [3] classify Inter Organizational Systems (IOS) for inter-company coordination (or collaboration), into three main types: vertical, horizontal and cross IOS. A horizontal IOS links a homogeneous group of organizations in order to foster their mutual cooperation. A horizontal IOS typically reflects a market coalition or partnership. A vertical IOS links organizations that play different roles in a value chain. Vertical IOS generally support the value chain of an IOS participant. The more unique roles the participants play, the higher the vertical linkage. A cross IOS is an IOS that is both horizontally and vertically linked. Cross IOS enable benefits resulting from vertical cooperation combined with resource-oriented incentives of horizontal cooperation.

Following Scholz-Reiter \& Höhn [7] we selected the definition of supply chain process defined by the SCORmodel [6]. "The Supply-Chain Operations Reference-model (SCOR) is the product of the Supply-Chain Council (SCC), an independent, not-for-profit, global corporation with membership open to all companies and organizations interested in applying and advancing the state-of-the-art in supply-chain management systems and practices." [6]. The SCOR-model is used in consultancy and scientific research 
to describe and structure the supply chain processes in detail.

The classification we develop is based on the supply chain linkages and the SCOR delivery and source processes. We solely focus on the Source (S) and Deliver (D) processes, since these connect to the processes of other companies in a different tier of the supply chain. Combining the two dimensions as discussed above provides us with a framework (see Figure 1) in which we distinguish seven basic types of agent applications for B2B:

- Agent based horizontal sourcing - Horizontal sourcing involves the inter-organizational coordination of demand by parties that perform similar activities and/or services. Companies may want to source together, because they jointly can achieve cost reductions because of a larger demand than they can realize independently.

- Agent based horizontal delivery - Horizontal delivery concerns the coordination of deliveries and opportunities for consolidation. Here, parties work together to obtain a higher performance in transportation or resource utilization (e.g. warehousing).

- Agent based vertical sourcing - In vertical sourcing, a buyer identifies potential suppliers, negotiates product price, service and quality elements.

- Agent based vertical delivery - Vertical delivery concerns the coordination of deliveries of one supplier in accordance with the requirements of its customers, furthermore it involves managing the interdependencies of the supplier and its customers.

- Agent based cross-organizational sourcing - Crossorganizational sourcing involves inter-organizational vertical coordination of deliveries and customer requirements in combination with inter-organizational horizontal coordination of demand.

- Agent based cross-organizational delivery - Crossorganizational delivery relates to the coordination of deliveries of multiple suppliers, where the deliveries need to be delivered at the same time and place.

- Agent based cross-organizational network - The crossorganizational network relates to the interorganizational coordination of activities of many different actors that are closely coupled and highly interconnected in a network. These interdependencies include both vertical and horizontal collaboration between the parties.

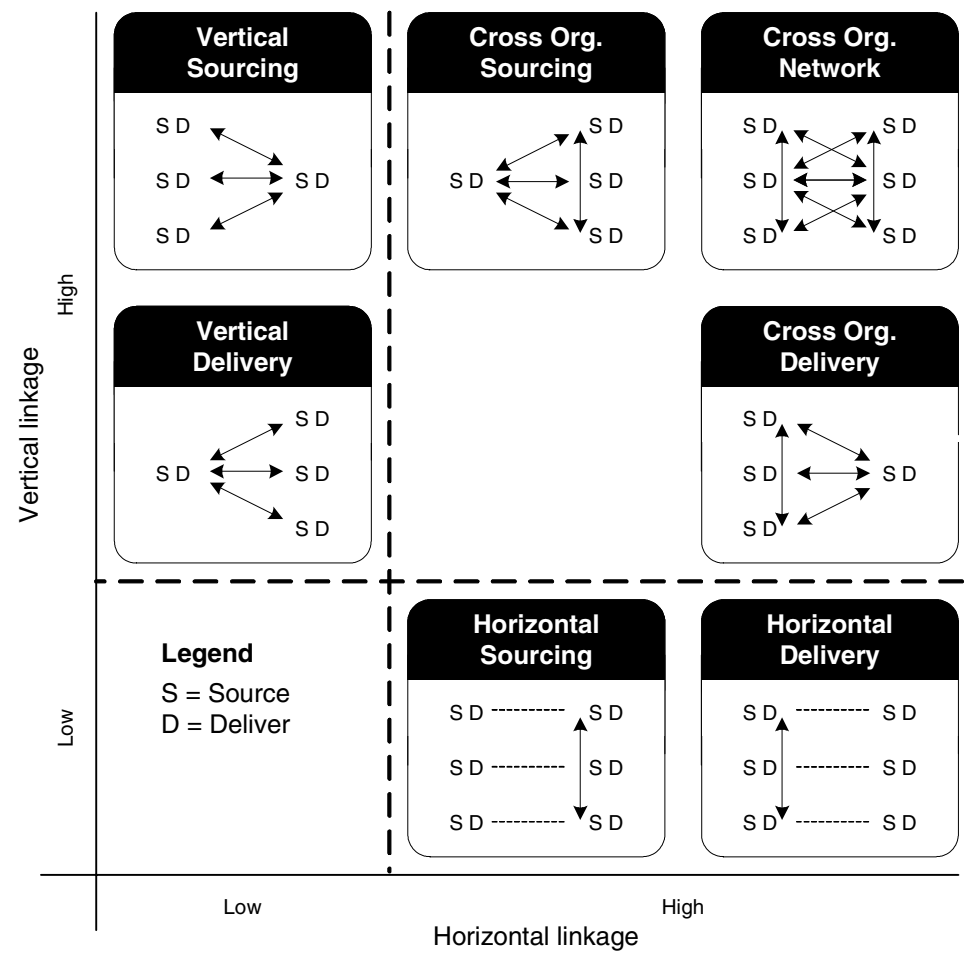

Figure 1: Classification of Agent-Based Collaboration Structures 
We initially tested and refined the framework by using it to classify a set of research and commercial B2B agent applications reported on in sources such as whitepapers, the Internet and Agent application conferences. Using the framework, we could easily position the examples found and create an overview of the current state of the field (see [2]). In the next section, we report on the use of the framework as a basis for a number of workshops. The objective was to identify the potential added value of Intelligent Agents in supply chains in practice. The next section summarizes the results of the workshops.

\section{Identifying potential for agents in supply networks}

To conduct the workshops, a seminar "Intelligent agents in supply chains, an exploration" was organized in August 2003. To goal of the seminar was to bring together academics, consultants, IT-specialists with the logistics industry specialist, to jointly identify potential promising applications of Intelligent Agent technology. As the logistics service providers and industries should be leading in adopting agent technology, their opinions were highly valued and the workshops were organized around their industry and supply chain types

A total of 120 company representatives were invited to attend to the seminar. Forty participants of thirty-four different companies or institutions participated. The attendees provided a well-balanced mix of the various groups (see Table 1). The seminar was subdivided into three sessions: a plenary session in the morning covering agent theory and sample applications, four parallel workshops to identify agent applications, and a concluding plenary session to exchange findings and discuss conclusions in the afternoon.

Table 1: Workshop attendance

\begin{tabular}{l|l}
\hline Industry & Number of companies \\
\hline Logistics & 9 \\
Shippers & 8 \\
ICT & 5 \\
Consultancy & 4 \\
Research institutes/universities & 7
\end{tabular}

Participants were asked to label areas were they saw most value. A total of 22 labels were put on the various areas of the framework. $22 \%$ of the labels were put in vertical sourcing and/or delivery relations in the supply chains. These participants expect that agent technology will contribute to further integration or collaboration of actors in their supply chain. $35 \%$ of the labels were put in the Cross organizational delivery and/or sourcing area's. According to these participants, horizontal or vertical sourcing is a first start, but the general expectation is that within five years, agent technology will develop towards agent based cross-organizational applications in one to many relations. A remarkable share of $30 \%$ of the labels was put on the cross-organizational network area. Overall, most potential of Intelligent agents was expected in the more complex cross organizational area's (65\%). A discrepancy in results between researchers, IT/consultants and participants from logistics and industry was observed. IT providers, researchers and consultants are more optimistic about adoption and implementation of complex cross-organizational future applications.

If the scores are measured without the IT providers', researchers' and consultants' opinions, $33 \%$ of the participating companies believe that vertical sourcing or delivery has most potential. Applications in horizontal sourcing or delivery and agent based cross-organizational network are less popular (e.g. 13\% and 20\%). The number of the participating companies that expect future applications in the cross-organizational sourcing or delivery field account for only 33\%, substantially less than the original $65 \%$.

Workshop participants were asked to label the most promising and feasible areas of the framework for their business and describe the agent application they had in mind. We summarize the results below.

\subsection{Horizontal sourcing}

Representatives from the fashion industry suggested that a very basic agent based IOS that would enable joined sourcing. Most stores are located in the heavily congested city centers. Daily, different trucks from different retailers are queuing in the same shopping streets. Joint sourcing would improve efficiency in transportation. An intelligent agents application could take care of the coordination and information exchange of shipments between the fashionindustry and select the best logistic provider. Eventually the agent application could take over transport planning and give transportation orders to the logistic providers (evolution to cross-organizational sourcing).

\subsection{Horizontal delivery}

A logistic provider gave an example of timely and complex coordination processes (tracking\&tracing, availability and planning and freight) with their subcontractors. Daily, this logistics provider uses more than one hundred sub-contractors, all having different IT systems. EDI, phone and fax are used in the communication and coordination process. Unfortunately different initiatives to make use of internet communication applications have failed. The added value of agents could be the realization of connectivity and information sharing with most of their sub-contractors.

\subsection{Vertical sourcing}

A food manufacturer expects that agent technology can 
contribute towards a better global sourcing process. In their view agents do not need much intelligence, but the added value is enclosed in realizing connectivity and (real-time) data collection (transparency in supplies). Future development should focus on adding more intelligence to support the sourcing process by giving product alternatives and eventually by giving sourcing advice.

\subsection{Vertical delivery}

A retailer currently organizes its logistics from two central distribution centers (DC). Currently, there is no clear indication on the time of arrival of suppliers. To optimize inbound and outbound logistics as well as warehouse management (planning staff, equipment, docks etc.), more information sharing with suppliers on arrivals is desired. The retailer sees agent technology as a tool that can meet this need. The capability of agents to realize connectivity and data-collection with the suppliers could provide a more efficient logistic process.

Participants of the copier industry see potentials for intelligent agent applications especially in the delivery of supplies. For example, the actual use of their copiers in the field is unknown. Agents can make it possible to integrate into the business process of a customer and to improve service. If an agent is able to identify when a machine is running out of supplies (toner), the manufacturer can anticipate by sending supplies. Intelligent agents make it possible to decrease inventory levels which is an advantage for the clients of the copier manufacturer (service tool).

\subsection{Cross-organizational sourcing \& delivery}

A logistics provider identified a potential multi-agent system to support transportation when demand fluctuates (promotions, season) and goods are not homogenous the capacity (volume) needs vary and the planning process is getting complex. Especially when information or transportation orders are received only a few days in advance. Agents can fulfil the need of information collecting/sharing (procurement or sales forecasts). The added value of intelligent agent technology will lead to a decrease of coordination and transportation costs (optimizing fleet planning).

\subsection{Cross-organizational network}

Several participating companies expect that within 5 years agent applications are acting in an agent based cross- organizational network. Unfortunately no examples of possible potential application were mentioned.

\subsection{Workshops conclusions}

In general, most participants were positive about the added value and the possible future applications agent technology could have in their supply chain(s). A variety of potential agent applications have been identified. Researchers, Consultants and IT vendors envision potential in cross organizational application areas while shippers and logistics/transport companies see most potential in vertical or horizontal applications. Several benefits of applying agents in these areas were identified, such as systems integration, optimizing planning, decentralized decisionmaking and supply chain visibility.

\section{Conclusions}

The main objective of this research was to identify potential opportunities for software agent technology to support current and future supply chains/networks. As none of the existing frameworks to address objective met our needs, we developed a framework based on SCOR and extending it to include horizontal, vertical and crossorganizational processes. The framework was successfully used as a basis for identifying promising areas in workshops with industry representatives. We present two sets of conclusions, first concerning the identified promising application areas and second regarding future research such as further applications of the framework.

Industry participants could successfully apply the framework to identify and describe potential applications of agent technology in their business. Industry had a preference for the horizontal and vertical coordination areas, whereas researchers and IT vendors/consultants identified most potential in the cross-organizational applications.

The framework developed in this research could serve as a tool to discover and position potential agent applications and assess their potential benefits based on comparable applications that exist in the same area. However, the framework currently only focuses on functional aspects of agent applications. It could be extended to allow the identification of critical success factors and to help anticipating value of the IOS. Several workshop participators noted that these aspects were important. Barriers such as personnel acceptance of new ways of working and the use of new technology, the willingness of supply chain partners to collaborate and adopt a common system were brought forward. Moreover, adoption depends on the current status of internal ICT systems. For example, a company may prefer to complete its ERP implementation before starting up an intelligent agent implementation. In addition, the need for clear business cases was expressed, including mechanisms to share costs and benefits of the agent system. Furthermore, in future research more detail should be added to the framework by including the detailed descriptions of B2B sourcing and delivery processes, as 
they are described in the SCOR model. Additionally a roadmap could be developed that lays out an evolutionary approach to the use of agents in supply chains for the coming decade. In the early phases, applications will mainly be developed that enable horizontal and vertical B2B processes in a single tier of a supply chain. In later phases, more complex projects could be initiated enabling cross-organizational process coordination potentially in multiple tiers of the supply chain, resulting finally in an increase of the number of agents that are active and alert on the information highway.

\section{References}

[1] Blake, M. B. (2002). B2B electronic commerce: Where do agents fit in? Proceedings of the AAAI-2002 Workshop on Agent Technologies for B2B E-Commerce, Edmonton, Alberta, Canada.

[2] Connekt (2003), "Intelligent Agents in supply chains, An explorative research", http://transport.connekt.nl/MGmain.html .

[3] Hong, I. B. and Kim, C. (1998). Toward A New Framework for Interorganizational Systems: A Network Configuration Perspective. Thirty-First Annual Hawail International Conference on System Sciences (Proceedings of HICSS 1998), Hawaii, IEEE Press.

[4] Khoo, L. P., Tor, S. B. and Lee, S. S. G. (1998). "The potential of intelligent software agents in the world wide web in automating part procurement." International Journal of Purchasing and Materials Management 1998 (January): 46-52.

[5] Nissen, M. E. (2001). "Beyond electronic disintermediation through multi-agent systems." Logistics Information Management 14(4): 256-275.

[6] SCC (2003). Supply-Chain Operations Reference Model - SCOR Version 6.0.

[7] Scholz-Reiter, B. and Höhns, H. (2002). Agent-based collaborative supply net management. Formal Proceedings of the APMS.

[8] Timm, I.J., P. Knirsch, H.-J. Müller, M. Petsch, N. Abchiche, P. Davidsson, Y. Demazeau, F. J. Garijo, O. Herzog, St. Kirn, C. Petrie, C. Tessier (2000) "Agent Technologies and Their Application Scenarios in Logistics"; Proceedings of the 14th European Conference on Artificial Intelligence, Berlin

[9] Wooldridge, M. and Jennings, N. R. (1995). "Intelligent agents: theory and practice." Knowledge management review 1995(January): 1-62.

[10] Yuan, Y., Liang, T.P., Zhang, J.J. (2001), "Using agent technology to support supply chain management: potentials and challenges". McMaster University, Michael G. DeGrootte School of Business, Working Paper Series no 453 